\title{
GLOBAL PATIENT SAFETY CHALLENGES: BREVE PANORAMA DOS AVANÇOS E PESQUISAS NO
}

\section{BRASIL}

Helen Cristiny Teodoro Couto Ribeiro - Enfermeira. Doutora em Enfermagem. Professora Adjunta do Curso de Enfermagem da Universidade Federal de São João del-Rei.

Após mais de uma década do lançamento do primeiro Global Patient Safety Challenge (Desafio Global para a Segurança do Paciente) da Organização Mundial da Saúde (OMS) e quatro anos do Programa Nacional de Segurança do Paciente (PNSP) do Ministério da Saúde no Brasil, o qual incorpora esses Desafios, a mudança de cultura com vistas à melhoria contínua da qualidade nos serviços de saúde tem avançado lentamente. As ações para o cumprimento destes Desafios variam de acordo com as regiões do país, as especialidades e os pontos da rede de atenção à saúde.

O primeiro Desafio Global "Clean Care is Safer Care", lançado em outubro de 2005, referiu-se às infecções relacionadas com a assistência à saúde (IRAS). Teve como pilar a higienização das mãos, uma ação simples e que ainda permanece como a primeira medida para redução das IRAS e da resistência antimicrobiana $^{(1)}$. Nesta área, o Brasil tem resultados de sucesso, como por exemplo, a segunda vitória consecutiva, de um hospital em São Paulo, no Prêmio Latino Americano de Excelência em Higienização das Mãos. Este Prêmio é uma iniciativa da OMS para reconhecer as instituições que demonstram melhoria da segurança dos pacientes, por meio da estratégia multimodal de higienização das mãos ${ }^{(2)}$. Contudo, em outros estados do país e em diferentes pontos da rede de atenção à saúde têm-se uma baixa adesão à higienização das mãos, falha na técnica correta e em todos os momentos recomendados pela OMS e alta taxa de IRAS ${ }^{(3-}$ 4). Os programas de higienização das mãos nos serviços de saúde alcançam resultados positivos após um curto período de tempo, sendo sempre necessárias novas abordagens para o aumento da adesão. Em um estudo realizado para identificar a abordagem dos protocolos de segurança do paciente instituídos pelo PNSP, que são também temáticas dos Desafios Globais, nas dissertações e teses elaboradas por enfermeiros e disponíveis no Catálogo da Associação Brasileira de Enfermagem (ABEN) do volume XIX (2001) ao XXXII (2013), apenas $13,21 \%$ dos 53 resumos analisados versavam sobre a higienização das mãos ${ }^{(5)}$. Neste horizonte, percebe-se que é necessário maior quantitativo de pesquisas que aborde esta temática. 0 primeiro Desafio Global "Clean Care is Safer Care" ainda precisa ser explorado na acadêmica, tanto na graduação quanto na pós-graduação e constar na agenda de gestores e profissionais de saúde.

O slogan "Safe Surgery Saves Lives" foi o segundo Desafio, proposto em janeiro de 2007, e contemplou os fundamentos e práticas da segurança cirúrgica no contexto da prevenção de infeç̧ões de sítio cirúrgico, anestesia e desenvolvimento de equipes cirúrgicas seguras e indicadores da assistência cirúrgica. Foi 
instituído também um checklist com 19 itens que se concentra na comunicação e práticas seguras em três períodos do fluxo natural de um procedimento anestésico-cirúrgico: antes da indução anestésica; antes da incisão cirúrgica da pele e antes do paciente sair da sala de cirurgia(6). A adesão e qualidade da aplicação deste instrumento variam nas especialidades cirúrgicas. Em um hospital de ensino na região Sul, dos 12.629 itens de verificação de 257 checklists da especialidade cirúrgica ortopédica avaliados, 91,5\% foram respondidos $^{(7)}$. Entretanto, estudo realizado em dois hospitais do Rio Grande do Norte, das 375 cirurgias urológicas e ginecológicas avaliadas, $61 \%$ tinham checklist, sendo que a adesão foi maior nas cirurgias ginecológicas, e a qualidade do preenchimento se relacionou com as cirurgias urológicas. Os autores deste estudo concluíram que é necessária uma implantação mais estruturada, visando assegurar a sua utilização adequada $^{(8)}$. Estes resultados demonstram que a utilização de checklist e sistemas de verificação na área da saúde, apesar de importante, ainda é uma prática recente nos processos de trabalho. No estudo que buscou analisar os caminhos percorridos pela enfermagem brasileira na área da segurança do paciente por meio da análise nas dissertações e teses elaboradas disponíveis no Catálogo da ABEN de 2001 a 2013, apenas 5,66\% dos estudos estavam inseridos no contexto do protocolo assegurar cirurgia em local de intervenção, procedimento e pacientes corretos ${ }^{(5)}$. Contudo, há evidências no Brasil de recorrentes e persistentes ocorrências de incidentes sem dano ou com dano (eventos adversos) na assistência cirúrgica ${ }^{(9-10)}$. Portanto, o Desafio Global "Safe Surgery Saves Lives" ainda constitui foco para melhoria na prática e campo de pesquisa a ser explorado.

Recentemente, em março de 2017, o terceiro Desafio Global "Medication Without Harm", foi lançado na Alemanha no evento Second Global Ministerial Summit on Patient Safety. Foram propostas soluções para a garantia da segurança do sistema de medicação desde a prescrição, transcrição, dispensação, administração até o monitoramento desta prática. O objetivo da OMS é reduzir no mundo $50 \%$ dos danos

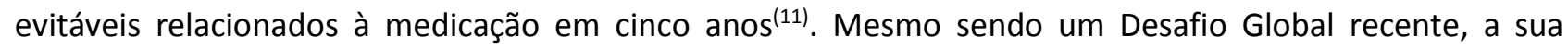
temática é alvo de trabalho de diversas instituições, ressalta-se o trabalho realizado pelos Conselhos Regionais de Enfermagem, Rede Brasileira de Enfermagem e Segurança do Paciente e Instituto para Práticas Seguras no Uso de Medicamentos do Brasil. Estas instituições têm proposto discussões, capacitações e elaborado documentos de instrução sobre a prática segura no uso de medicamentos. A temática que o envolve o sistema medicamentoso é amplamente pesquisada, tanto por enfermeiros quanto por outros profissionais, como os farmacêuticos. No estudo sobre dissertações e teses disponíveis no Catálogo da ABEN de 2001 a 2013 que abordam os protocolos de segurança do Ministério da Saúde, a temática do Desafio "Medication Without Harm" foi a mais pesquisada entre os três Desafios Globais para a Segurança do paciente. Dos 53 trabalhos avaliados, 20,75\% das dissertações e 2,77\% das teses versaram sobre o protocolo uso e administração de medicamentos ${ }^{(5)}$. Este quantitativo expressivo de pesquisas, se comparado aos resultados dos demais Desafios Globais, pode ser devido à grande responsabilidade que é transferida para a equipe de enfermagem pelo fato de sua atuação no preparo e na administração de medicamentos. Muitos erros não detectados nas etapas de prescrição, transcrição e dispensação são atribuídos à enfermagem, por 
ser ela, na maioria das vezes, a última barreira para interceptar e evitar um erro de medicação. Desta forma, os profissionais de enfermagem devem fazer deste Desafio Global uma oportunidade de discussão de seus processos e condições de trabalho dentro do sistema de medicação, com vista a oferecer maior segurança para os pacientes. Por fim, espera-se que as ações propostas pelo recente Desafio Global "Medication Without Harm" sejam assumidas pelos gestores, compreendidas e implementadas pelos profissionais que atuam na prática assistencial e cobradas pelos conselhos de saúde e cidadãos que recebem a prestação de serviços em todos os níveis e pontos da rede de atenção à saúde, pública ou privada do Brasil.

Para além do cumprimento dos Global Patient Safety Challenges, o progresso na área da segurança do paciente ocorre de forma gradual. A assistência prestada pelos serviços de saúde continua de baixo grau de confiabilidade, o que significa que os pacientes frequentemente sofrem danos que poderiam ter sido prevenidos $^{(12)}$. Para o progresso da qualidade da assistência e, especificamente para o avanço do domínio da qualidade "segurança do paciente", é necessário um movimento amplo no Brasil. É fator crítico de sucesso o envolvimento de todos os profissionais de saúde, independente da especialidade, região do país e nível ou ponto de atenção que atuam. Quase a totalidade dos estudos referentes aos protocolos de segurança do paciente brasileiros disponíveis no Catálogo de dissertações e teses da $A B E N^{(5)}$, teve como cenário o ambiente hospitalar. Faz-se necessário expandir as estratégias para melhoria da segurança do paciente para os demais serviços/pontos da rede de atenção à saúde. A qualidade em suas diversas dimensões, e especificamente a dimensão segurança do paciente, proporcionada em um ponto de atenção refletirá a assistência de qualidade no outro ponto.

Além disso, a participação ativa do paciente na mitigação de incidentes é indispensável, é necessário empoderá-los para isso, uma vez que estes são os mais interessados em sua própria segurança. Ademais, é necessária mudança de paradigma, de uma cultura de punição para uma cultura de aprendizado frente aos incidentes que ocorrem na assistência à saúde.

\section{Referências}

1- WHO. World Health Organization [Internet]. Global Patient Safety Challenge: Clean Care is Safer Care. Geneva, Switzerland: 2005 [citado em 2017 Abr 30] Disponível em: http://www.who.int/patientsafety/events/05/BriefingNoteEnglish.pdf?ua=1

2- Portal Hospitais Brasil [Internet]. Hospital brasileiro conquista pela segunda vez o Prêmio Latino Americano de Excelência em Higienização das Mãos [citado em 2017 Abr 30] Disponível em: http://portalhospitaisbrasil.com.br/hospital-brasileiro-conquista-pela-segunda-vez-o-premio-latinoamericano-de-excelencia-em-higienizacao-das-maos/

3- Borges LFA, Rocha LA, Nunes MJ, Gontijo Filho PP. Low Compliance to Handwashing Program and High Nosocomial Infection in a Brazilian Hospital. Interdisciplinary Perspectives on Infectious Diseases. 2012:1-5. [citado em 2017 Abr 30]. Disponível em: https://www.hindawi.com/journals/ipid/2012/579681/ 
4- Oliveira $A C$, Paula AO, Souza MA, Silva AG. Adesão à higiene de mãos entre profissionais de um serviço de pronto atendimento. Revista de Medicina. 2016 Dez 95(4):162-67. [citado em 2017 Abr 30]. Disponível em: http://www.revistas.usp.br/revistadc/article/view/122861/122463

5- Gomes ATL, Salvador PTCO, Rodrigues CCFM, Silva MF, Ferreira LL, Santos VEP. A segurança do paciente nos caminhos percorridos pela enfermagem brasileira. Rev. Bras. Enferm. 2017 Feb 70(1): 146-54. [citado em 2017 Abr 30]. Disponível em: http://www.scielo.br/scielo.php?script=sci arttext\&pid=S003471672017000100146\&lng=en. http://dx.doi.org/10.1590/0034-7167-2015-0139

6- WHO. World Health Organization [Internet]. Safe Surgery Saves Lives. Geneva, Switzerland: 2008 $\begin{array}{llllll}\text { [citado em } & 2017 & \text { Abr } & 30] & \text { Disponível }\end{array}$ http://apps.who.int/iris/bitstream/10665/70080/1/WHO IER PSP 2008.07 eng.pdf

7- Amaya MR, Maziero ECS, Grittem L, Cruz EDA. Análise do registro e conteúdo de checklists para cirurgia segura. Escola Anna Nery. 2015 Abr;19(2):246-51. [citado em 2017 Abr 30] Disponível em: http://www.scielo.br/pdf/ean/v19n2/1414-8145-ean-19-02-0246.pdf

8- Freitas MR, Antunes AG, Azevedo BNL, Fernandes FC, Monte LC, Gama ZAS. Avaliação da adesão ao checklist de cirurgia segura da OMS em cirurgias urológicas e ginecológicas, em dois hospitais de ensino de Natal, Rio Grande do Norte, Brasil. Cad Saúde Pública. 2014; 30(1):137-48. [citado em 2017 Abr 30]. Disponível em: http://www.scielo.br/pdf/csp/v30n1/0102-311X-csp-30-01-00137.pdf

9- Moura MLO, Mendes W. Avaliação de eventos adversos cirúrgicos em hospitais do Rio de Janeiro. Rev. Bras. Epidemiol. 2012; 15:523-35. 2012. Disponível em: http://www.scielo.br/scielo.php?script=sci arttext\&pid=S1415-790X2012000300007

10- Paranaguá TT, Bezerra AL, Silva AE, Azevedo Filho FM. Prevalência de incidentes sem dano e eventos adversos em uma clínica cirúrgica. Acta Paul Enferm. 2013; 26(3):256-62. [citado em 2017 Mai 10] Disponível em: http://www.scielo.br/pdf/ape/v26n3/09.pdf

11- WHO. World Health Organization [Internet]. Medication Without Harm: WHO's Third Global Patient Safety Challenge. Geneva, Switzerland: 2017 [citado em 2017 Abr 30] Disponível em: http://www.who.int/patientsafety/medication-safety/en/

12- NPSF. National Patient Safety Foundation [Internet]. Free from Harm: Accelerating Patient Safety Improvement Fifteen Years after To Err Is Human. Boston, MA: 2015 [citado em 2017 Abr 30] Disponível em: http://c.ymcdn.com/sites/www.npsf.org/resource/resmgr/PDF/Free from Harm.pdf 